\section{EL PROBLEMA DEL TIEMPO EN LA FÍSICA: UN PLANTEAMIENTO EXISTENCIAL DE LA CIENCIA DESDE HEIDEGGER}

\author{
Felipe Johnson \\ Universidad de La Frontera, Temuco/Chile \\ felipe.johnson@ufrontera.cl
}

\section{THE PROBLEM OF TIME IN \\ PHYSICS: AN EXISTENTIAL APPROACH TO SCIENCE FROM HEIDEGGER'S POINT OF VIEW}

Cómo citar este artículo/Citation: Johnson, F. (2016). “El problema del tiempo en la física: un planteamiento existencial de la ciencia desde Heidegger". Arbor, 191 (775): a294. doi: http://dx.doi.org/10.3989/arbor.2016.777n1010
Copyright: (c) 2016 CSIC. Este es un artículo de acceso abierto distribuido bajo los términos de la licencia Creative Commons Attribution-Non Commercial (by-nc) Spain 3.0.
RESUMEN: Este artículo se propone caracterizar el sentido propio del ejercicio científico desde nociones del pensamiento de Heidegger como la caída y la actitud teórica. En la búsqueda de una vía de acceso que permita dicha tarea, se indagará en la relación que ha tenido la física con el concepto de tiempo. Este será el punto de partida desde el cual se advertirá que su trato particular con el tiempo devela rasgos propios de su modo concreto de ocuparse con la realidad. Así, este artículo planteará cómo es que la concepción de tiempo clásica y sus reformulaciones se fundan, en última instancia, en una relación ocupada del existir que caracterizaría el quehacer científico mismo. Con ello, se realizará una elucidación de la ciencia que rinda cuenta del presente como el horizonte existencial de su despliegue fáctico.

PALABRAS CLAVE: tiempo lineal; presente; caída; ciencia; Heidegger.
ABSTRACT: This article aims to characterise the meaning of scientific practice deriving from Heidegger's conceptions of falling and the theoretical attitude. To do so, we will inquire into the relationship that physics has with the concept of time. This starting point reveals how his particular treatment of time unveils specific features of how own way of understanding our encounter with reality. Thus, this article shows that the classic concept of time and its reformulations are based, in the final analysis, on an occupied relationship of existence that characterises the scientific know-how in general. Considering the above, this elucidation of science will give account of the present as the existential horizon of its factual unfolding.

KEYWORDS: linear time; present; falling; science; Heidegger. 


\section{EL PROBLEMA DEL TIEMPO EN LA CIENCIA}

Toda investigación científica cuyo objeto de estudio se centre en algún tipo de ente del mundo requiere del tiempo. Ya sea como parámetro implícito o como medida explícita, en cualquier estudio, el pasado, el presente y el futuro delimitan determinados modos de estudio tanto del hombre individual, de las comunidades humanas, como de la naturaleza en general. Cabe mencionar a la historia, i.e., la investigación de hechos humanos colectivos pasados. También hallamos determinadas teorías psicológicas, como las psicoanalíticas, o biológicas, como las evolucionistas, las cuales se detienen a investigar interacciones ya acontecidas, entendiéndolas como factores complejos que contribuyen a explicar estados anímicos u orgánicos presentes. Se advierte, a la vez, en tales investigaciones una particular pretensión predictiva que les conduce a adelantarse a episodios futuros, mediante la aclaración del pasado y del presente. En definitiva, pasado, presente y futuro aparecen como el horizonte fundamental de la investigación científica. El concepto de tiempo, podríamos decir, es un concepto fundante de las ciencias humanas y de la naturaleza. No es, por tanto, una tarea ociosa preguntarnos por la noción de tiempo que subyace al quehacer científico, cuando dicha comprensión parece determinar su propio desarrollo. Precisamente, este es el motivo del presente trabajo: mediante una aclaración de la noción tradicional del tiempo, interrogar por la esencia del ejercicio científico.

No todas estas investigaciones, sin embargo, parecen haber hecho del tiempo un problema explícito. Quizás sea la física aquella disciplina en la cual es posible hallar una confrontación manifiesta con él. Su historia rinde cuenta de determinadas explicaciones que conducen incluso a desvincular ciertos fenómenos físicos de su necesaria dependencia con el tiempo. En efecto, el tiempo experimentado en la observación natural, entendido como el avance progresivo e inalterable de instantes, pareciera no aplicarse a fenómenos moleculares. Sobre esto nos enseña el fenómeno de la entropía. En el caso del derrame de un fluido, por ejemplo, una determinada masa muestra en principio un cierto orden, no obstante, las moléculas que componen la masa total del líquido se expanden en el espacio sin orden aparente alguno. La masa física se desordena molecularmente, y dicho desorden, observable solo como "derrame caótico del líquido", podría permitirnos determinar el pasado y el futuro del evento observado. La pregunta que el físico se haría (cf. Hacyan, 2004, p. 73) es si acaso, a nivel molecular, la trayectoria que recorre una sola de esas moléculas rinde cuenta efectiva de un progreso de pasado $a$ futuro, como lo hace el cambio observable de la totalidad del fluido. Y es que atendiendo al movimiento de una sola de ellas, ya sea progresiva o regresivamente en el tiempo, lo que puede ser constatado es el mero recorrido de trayectorias a la manera de choques aleatorios, fenómeno que parece ser indiferente a la dirección temporal en la que se le observe. Con estas observaciones, la clásica comprensión del tiempo, como una instancia necesariamente vinculada al cambio de lo estudiado, parece volverse completamente cuestionable. El tiempo pasa a ser una propiedad que se daría solo en ciertos niveles de la observación humana, mas no pareciera ser un parámetro de medida absoluto.

Asimismo, la continuidad inmodificable del progreso del tiempo, es decir, su ritmo de avance, parece ser otro aspecto cuestionado por la física actual. La teoría de la relatividad parece, de hecho, objetar la invariabilidad de dicha continuidad. El tiempo parece expandirse o contraerse de manera inversamente proporcional a la proximidad que posea el sistema referencial respecto de la velocidad de la luz, por lo cual, no mostraría un ritmo invariable, como lo sostuvo la física newtoneana. Por ende, se ha debido fijar otro parámetro absoluto de referencia que permita realizar el cálculo de la interacción de los fenómenos de la realidad. En el caso de Einstein, dicho criterio de referencia absoluto sería la velocidad de la luz.

Como se advierte, el cuestionamiento de dos características del tiempo tan decisivas como son su vinculación necesaria con el movimiento y el cambio, y su inalterable ritmo de avance, parecen indicar que su definición ha de ser necesariamente reformulada. Sin embargo, antes de decidir si tales reformulaciones implican un efectivo progreso respecto de una comprensión del tiempo mismo, estimamos que lo decisivo es detenernos en el sentido de estas. Así, sería conveniente señalar que los intentos de reelaborar la noción de tiempo por parte de la física parecen motivarse desde el interés por afianzar parámetros de medida, requeridos por la tarea de calcular fenómenos que superan los límites de la observación natural humana, ya sea a un nivel molecular como astronómico. Sin embargo, mientras la física intenta definir nuevamente el concepto de tiempo, reaccionando frente a su noción tradicional, no parece ser necesaria para ella la pregunta metódica que podría contribuir a una aclaración del tiempo mismo, esto es, la pregunta por la manera cómo sería posible acceder a dicho fenómeno para comprenderlo en cuanto tal. Este es justamente 
el índice que, a nuestro juicio, señala hacia una problemática aún más decisiva y que es la que pretendemos revisar en el presente trabajo. El modo como la física ha tratado el problema del tiempo conduce a detectar que las nuevas definiciones que esta realice del mismo atienden a determinadas motivaciones que rigen su propio ejercicio. Así, una aclaración de la relación de la física con el tiempo podría llevar a entender y a caracterizar el propio sentido de su investigación. Por tanto, a partir de dicha relación es que quisiéramos encontrar una vía para esclarecer en qué consiste fundamentalmente el quehacer científico en general.

Sin embargo, antes de comenzar, es importante destacar que las presentes discusiones se proponen abordar el problema del tiempo y del sentido íntimo del quehacer científico desde los planteamientos existenciales de Martin Heidegger. Desde ellos, nuestra pretensión es la de abordar el ejercicio científico haciendo énfasis en aquella relación vital que se advierte precisamente cuando atendemos al modo como resulta la comprensión del fenómeno del tiempo en tanto que progresivo y lineal: la relación científica. En dicho contexto, será necesario, entonces, reparar en la recepción de la noción de tiempo en la física, entendiéndola como un modo paradigmático de confrontación teórica con el problema del tiempo en general. De esta manera, desde el pensamiento de Heidegger se advertirá que el tiempo en su sentido progresivo y lineal pareciera acontecer tras una modificación de una experiencia cotidiana del mismo que se hace observable en la problemática de la significatividad del tiempo descrita por el mismo Heidegger. Así, será posible advertir que la concepción general de tiempo en la ciencia no deviene sino desde una des-vivificación, es decir, de una reducción de caracteres vitales pertenecientes a la vivencia del tiempo cotidiano, que resultan finalmente a partir de una condición del existir mismo, a saber, de su condición caída. Abordando, pues, el tiempo lineal desde las nociones heideggerianas se observará que tal caída, en tanto actitud inmediata del existir con su entorno, no parece radicar sino en una ocupación con lo que se le muestra efectivamente ahí delante, y que esta acontece, por lo demás, en un modo temporal específico como es el presente. En definitiva, las reflexiones mostrarán cómo es que la caída y su respectivo privilegio del presente serían aquellos momentos existenciales fundamentales para entender el sentido íntimo del ejercicio científico en general, desde los cuales parecen concebirse conceptos como el de este trabajo, a saber, el del tiempo tal y como aún parece predominar en la consideración científica.
No obstante, como primer paso, será preciso delimitar el concepto tradicional de tiempo en juego. Para ello, atenderemos a los puntos importantes de la reconstrucción que realiza Heidegger de la noción de tiempo aristotélica, pues, en ella se encuentra un planteamiento sistemático de los elementos que constituyen su comprensión clásica. Esta primera discusión nos proporcionará los elementos necesarios para abordar el problema de la ciencia y su relación con el tiempo en términos existenciales. ${ }^{1}$

\section{EL CONCEPTO TRADICIONAL DE TIEMPO DESDE LAS LECTURAS HEIDEGGERIANAS DE ARISTÓTELES}

Detengámonos en la definición del tiempo expuesta por Aristóteles en Física, a saber, en cuanto "número del movimiento según el antes y el después" (IV a. C./1956, p. 219b2). Dicha definición será el punto de partida de la interpretación heideggeriana, tal y como se encuentra en su lección Die Grundprobleme der Phänomenologie.

Que el tiempo sea el "número del movimiento" ya es un índice para entender que este solo se manifestaría cuando hay movimiento. No habría tiempo sin movimiento, o, en términos amplios: "sin cambio (metabolé) no hay tiempo" (Aristóteles, IV a. C./1956, p. 218b23). El tiempo, precisa Heidegger (1975/1997, p. 337), no podría ser percibido, entonces, como una propiedad del objeto mismo que se mueve, pues, no sería algo que se presenta junto a su peso, su color, etc. Por lo cual, el tiempo sería más bien un fenómeno que se vincularía al movimiento del móvil y no al móvil en movimiento. Pero, igualmente, el tiempo no podría ser identificado con el movimiento mismo (kínesis), ya que precisamente es "algo del movimiento" (kinéseos ti). Así se advierte que el tiempo ha de darse separado tanto del objeto como del movimiento mismo, ya que sería, ante nada, el número del movimiento.

No obstante, el sentido de ser número es lo que ahora debiera ser precisado, y para esto Heidegger recurrirá al vocablo griego "arithmós" en relación con la voz griega en participio medio-pasivo "arithmoúmenon" (Aristóteles, IV a. C./1956, p. 219b8), es decir, ser contado o "lo contado" (Gezähltes) (Heidegger, 1975/1997, pp. 338 ss.). En palabras de Heidegger: "El tiempo como número del movimiento es lo contado en (an) el movimiento" (1975/1997, p. 339). Quisiéramos destacar la preposición alemana "an" que figura en la reconstrucción del texto aristotélico, pues, subyace en ella una decisión interpretativa importante. A diferencia de la misma, podemos encontrar casos en los cuales 
se le traduce conservando el sentido genitivo del vocablo "kinéseos". Para ilustrar lo anterior podemos mencionar a Brentano, quien traduce "Zahl der Bewegung" (1862/1960, pp. 134 ss.) o Fink, quien opta por: "Anzahl der Bewegung" (1957, p. 226). Asimismo, en francés, Aubenque nuevamente conserva el sentido posesivo del genitivo: "nombre du mouvement" (1962, p. 50). Sin embargo, mientras que el posesivo "de" parece dirigir la atención a la vinculación que habría entre el tiempo y el movimiento, la inclusión de la preposición "an" parece subrayar cierta independencia entre ambos. De esta manera es que la lectura heideggeriana revela una autonomía que quisiéramos fijar como primer carácter del tiempo aristotélico. Dicha autonomía del tiempo, no obstante, nos conducirá a otra caracterización fundamental del mismo.

Para esto, Heidegger deberá detenerse en la estructura misma del movimiento (kínesis). Según lo expone, su carácter general ha de ser el cambio (metabolé) (cf. Heidegger, 1975/1997, p. 343), por tanto, este viene a ser su propia estructura. Así, en cuanto "metabolé" radica en un "ek tinos eis ti" (Aristóteles, IV a. C./1956, p. 224b1), i.e., en un "tránsito desde algo $a$ algo", el traslado espacial (forá), por ejemplo, puede comprenderse como el "tránsito desde un lugar $a$ otro". Sin embargo, el sentido espacial que parece poseer dicho traslado no puede ser un carácter esencial del movimiento (kínesis) mismo. Todo traslado es, por cierto, movimiento, pero no todo movimiento es traslado, por lo cual, solo en cuanto traslado es posible advertir en él este sentido espacial. En otro tipo de movimiento, como la alloíosis, es decir, en la alteración de una propiedad en un objeto, se observa lo anterior. El cambio del color azul, por ejemplo, al verde, es un movimiento e implica, por ende, un tránsito, en este caso "desde el azul al verde", pero el color no se traslada de lugar, más bien "se vuelve otro" (cf. Heidegger, 1975/1997, p. 343). En otras palabras, la estructura del cambio, propia del movimiento, como "tránsito desde algo a algo", carece primariamente de sentido espacial, aunque presenta un carácter más decisivo aún, a saber, una determinada "prolongación" (Dehnung), i.e., una "extensión" (Erstreckung) (cf. Heidegger, 1975/1997, p. 343).

Este "tránsito desde... a...", ya sea en el sentido del "traslado desde un punto A a uno B", o la "alteración desde el azul al verde", implica, entonces, ser una extensión ininterrumpida. Lo que caracteriza al movimiento en cuanto tránsito es su ejecución de una sóla vez "desde... a...". De ahí que Aristóteles advierta en él el carácter de synechés, o, de acuerdo con la traducción de Heidegger, de un "estar-contenido-en-sí" (Insich-zusammengehaltenes) (Heidegger, 1975/1997, p. 344). Se trata, así, de lo "con-tenido", de un continuum, esto es, de un "constante" (Stetiges). Del mismo modo, se advierte el carácter de mégethos, i.e., "tamaño", destacado por Aristóteles (cf. IV a. C./1956, p. 219a10), ya que el movimiento en cuanto "tránsito desde... a..." se prolonga, se extiende, adquiere una dimensión, constituida por lo que en dicho tránsito se encuentra constante.

Entendiendo al movimiento, pues, desde su extensión no espacial y continua podemos ahora señalar cómo es que el tiempo puede ser "lo contado" (arithmoúmenon). Aristóteles dice: "el tiempo sigue al movimiento" (IV a. C./1956, p. 219b23). El sentido de "seguir" (akoloúthein) en la cita vendría a caracterizar de manera más precisa la autonomía del tiempo respecto del movimiento que hemos destacado. Dicho "seguir" no mienta un "resultar de...", como si debiera acontecer primero el movimiento para que, luego, el tiempo se generase. El tiempo, más bien, se sigue del movimiento en el sentido en que cuando se percibe de una vez el movimiento, el tiempo viene a ser, en su prolongación, co-percibido. El tiempo se hace co-presente junto al tránsito "desde... a..." ¿Cómo se co-presenta, pues, el tiempo en la percepción instantánea de este "tránsito desde... a..."? En cuanto un "antes" (próteron) y un "después" (hýsteron), según reza su definición. En el caso del traslado espacial, explica Heidegger, el movimiento se expresa como el fenómeno que se prolonga desde el punto A al B, es decir, como un "desde aquí (von dort her) hacia allá (dort-hin)" instantáneo, percepción en la cual, "reteniendo" "lo de antes" (Voriges) y "esperando" "lo que venga después" (Nachheriges), se hace posible "intuir" el tránsito de una sola vez (cf. Heidegger, 1975/1997, p. 347). En otras palabras, toda percepción de tránsito, o sea, del movimiento, requiere de la co-percepción de lo que hubo en términos de un "antes" y de lo que habrá, en términos de un "después". Sin los índices "antes" y "después", el fenómeno del "tránsito" en cuanto tal no podría ser aprehendido.

Lo "contado" del movimiento, por lo tanto, se expresaría cuando decimos "antes allí", "ahora acá", "después allá", de modo que cada lugar que un móvil recorriera se traducirá, dice Heidegger, en un "ahora ahí, ahora ahí, ahora ahí (Jetzt-dort)" (1975/1997, p. 347). Estos consecutivos "ahora" son precisamente lo contado (arithmoúmenon) cuando el movimiento es 
percibido como "tránsito desde... a...". Así, el tiempo es aquello que se cuenta, al modo de diversos "ahora" respecto de un "antes" y un "después", y esto ocurre junto con la percepción del tránsito mismo. El tiempo, en definitiva, no es el móvil, ni tampoco el movimiento del móvil, es un fenómeno "adyacente" a ellos.

He ahí la razón por la cual Aristóteles, a juicio de Heidegger, decidiría caracterizar al tiempo como número. Se trata de aquella autonomía que hemos destacado, enfatizando en que el tiempo sería un fenómeno "adyacente" al movimiento. En este sentido, Heidegger precisará que el tiempo para Aristóteles no es límite (péras) (cf. Heidegger, 1975/1997, p. 352s.) de lo que en él ocurre, ya que en estricto rigor no es ni principio ni fin del movimiento, mucho menos del ente. El ahora en el que ocurre un movimiento no acaba con el movimiento mismo, sino que se relaciona al tiempo que vendrá después. Por lo tanto, es el movimiento el que tiene un comienzo y un fin, mientras que el tiempo sigue su propio curso. Pues bien, si se dice "diez caballos", siguiendo el ejemplo de Heidegger, nada se afirma respecto a los caballos mismos. El modo de ser del número radica en aparecer junto a algo, mas no incide en este respecto ni de su contenido, ni de su manera de ser, ni de su movimiento como tal (cf. Heidegger, 1975/1997, p. 353). Así, la decisión aristotélica de caracterizar al tiempo como número, implicaría, en última instancia, enfatizar en él su co-presencia autónoma respecto al movimiento. Según dicha autonomía, entonces, el tiempo abarcaría, comprendería (Umhalten) al movimiento. El tiempo es lo que, en su autonomía, contiene lo que acaece dentro de él, mientras que el movimiento viene a ser entendido como lo "contenido" por este (cf. Heidegger, $1975 / 1997$, p. 356). Se trata de dos instancias juntas, pero esencialmente separadas.

Entonces bien, mediante las precisiones de la lectura heideggeriana de Aristóteles, el tiempo se muestra primariamente como una instancia autónoma. Por otra parte, este parece radicar en la fijación de diversos "ahora" que vienen a ser lo contado del movimiento en relación a un antes y un después. Este carácter extenso del tiempo implica, por último, contener, mas no incidir, en el movimiento que en él ocurra. De esta manera, la siguiente tarea será comprender cómo es que la ciencia ha asumido esta noción de tiempo, pero, a la vez, cómo es que en ella dicha noción se modifica. Precisamente, dicha modificación habrá de señalar el modo propio como las ciencias mismas se despliegan.

\section{LA FÍSICA Y SU DEFINICIÓN DEL TIEMPO AL SERVI- CIO DEL MOVIMIENTO Y EL CAMBIO}

Como ya decíamos, la física ha sido aquella ciencia que explícitamente se ha confrontado con el concepto tradicional de tiempo. Su noción clásica radica en que este avanza en la secuencia "pasado", "presente" y "futuro" y estos tres modos temporales son entendidos como los escenarios donde respectivamente acontecieron, acontecen y acontecerán hechos reales. El tiempo se concibe como un flujo lineal constante que progresa unidireccionalmente y en el que se contienen los diversos eventos de la realidad. Así, consecución, unidireccionalidad y ser el continente de hechos, son rasgos fundamentales de la definición tradicional de tiempo en la física.

Así formulado, este tiempo pareciera identificarse con aquel postulado por Aristóteles. No obstante, no coinciden totalmente. Esto se debe fundamentalmente a las pretensiones que subyacen al propio ejercicio de la física. Cabe destacar que el tiempo en esta adquiere sus primeras expresiones en un área de estudio muy particular: la cinemática (cf. García Doncel, 1989, p. 41). En dicho contexto, el tiempo se integra en una disciplina cuya pretensión primaria es la de atender a un fenómeno del mundo perceptible, esto es, el movimiento local. De esta manera es que, en un comienzo, el tiempo se determina mediante el movimiento constante del sol. Luego, en el S. XVII, Galileo igualmente se sirve del movimiento para la medición del tiempo, pero, esta vez, pesando pequeños vasos que progresivamente se irán llenando con el agua conducida desde una cubeta (cf. García Doncel, 1989, pp. 42 ss.; Galilei, 1638/1898, p. 213). Los pesos de las cantidades de agua vendrían a indicar el tiempo del movimiento de un móvil que se dejaba caer simultáneamente sobre un plano inclinado. Por tanto, el objeto de interés para Galileo y, en general, para la cinemática, no es el tiempo, sino la aceleración del móvil que cae describiendo una determinada trayectoria. Así, los valores temporales establecidos vendrían a radicar, ante nada, en el número que se obtiene del flujo del agua que cae. Con ello se constata, en consecuencia, una identidad asumida en tal investigación entre el movimiento constante y el tiempo, pues, de lo que se trata, en última instancia, es de fijar un parámetro estable para medir la aceleración del movimiento contingente de la Naturaleza.

Como se advierte, entonces, el movimiento vendría a rendir cuenta de dos características fundamentales del tiempo. Por una parte, el movimiento uniforme, ya sea de los astros como del agua, son comprensi- 
bles como parámetros inalterables del devenir terrestre. Por otra parte, el tiempo es, a la vez, comprendido como una dimensión paralela al mismo. En un momento pudo haber sido el desplazamiento constante del sol, pero también pudieron ser otros astros, como ocurrió con las "Estrellas Mediceas", aquellos cuatro satélites que Galileo descubriría en Júpiter y que describirían "períodos de oscilación perfectamente definidos" (García Doncel, 1989, p. 45). Como fuere, el tiempo viene a ser entendido en autonomía respecto del cambio y la corrupción sublunar, pues, describe un flujo imperturbable y retraído en sí, el mismo que Newton determinará, por cierto, como el "tiempo absoluto", contraponiéndolo al "tiempo relativo", que es el de la Naturaleza.

En este contexto, la identificación del tiempo con el movimiento parece rendir cuenta de dos de sus caracteres fundamentales: su constancia y autonomía. No obstante, un tercer carácter, su unidireccionalidad, atiende a otro problema con el cual la física se confrontaría: la causalidad (cf. Reiser, 1926, p. 238). Podríamos afirmar que el cambio que presenta la realidad no es un fenómeno espontáneo, sino, más bien, un resultado. En efecto, todo cambio ha de ser explicado por un evento anterior que lo posibilite. Se trata de la causa eficiente discutida por Aristóteles en Metafísica (IV a. C./1957, p. 1013b), en cuanto "de dónde" (hóthen) del movimiento (kínesis), y que luego se consolidará como el principio para comprender los acontecimientos que ocurren en el mundo físico. En Descartes encontramos esta causalidad formulada de la siguiente manera: "Debe haber por lo menos tanta realidad en la causa eficiente y total como en su efecto" (1641/1996, p. 32). Así, en la realidad observada podemos entender que las características que se encuentren en un determinado objeto han debido provenir ya de una instancia anterior, desde la cual ella ha "heredado" parte o la totalidad de las propiedades que exhibe. La causa, en consecuencia, ha de ser necesariamente anterior al efecto, ya que desde ella sería este de facto lo que es. En vistas a lo dicho, se harán necesarios los parámetros del "antes" y "después" en aquel movimiento constante e invariable que parece ser el tiempo, justamente para ordenar cronológicamente los hechos y así distinguir con exactitud las causas de los efectos. La serie del tiempo, por ende, deberá avanzar, jamás retroceder, ya que lo que interesa es la detección de "lo ocurrido", en vistas a una comprensión del estado actual del cambio.

Ocupada la física con el movimiento y el cambio alterables de la Naturaleza, el concepto de tiempo autó- nomo, alejado de todo devenir terrestre, constante y unidireccional, parece serle completamente útil. Pero este no siempre será el caso. Un estadio importante para comprender esto lo representa el positivismo de Mach. Su posición, empero, no estaba sino motivada por radicalizar el estatus de realidad de lo observable, por lo cual, nociones universales perderán su dignidad ontológica y deberán establecerse como productos de ciertos estados fisiológicos. Esta es precisamente su intención al caracterizar al tiempo como el resultado de una operación abstracta como sería la de traducir en número una sensación concreta como puede ser un movimiento percibido mediante la rotación de un astro o de las manecillas de un reloj (cf. Mach, $1885 / 1922$, p. 285). Por lo tanto, la idea de un concepto de tiempo "real" no sería más que una universalización, ilegítima, por lo demás, pues, carecería de todo sustento sensible (cf. García Doncel, 1989, p. 52). Con ello, no solo se observa la ya destacada autonomía del tiempo respecto del movimiento terrestre, sino que, en este caso, se le reduce al sujeto, y se le arrebata su existencia efectiva. El tiempo sería desplazado de la realidad para volverse dependiente del observador individual. Por su parte, Faraday rendía cuenta de la acción a distancia del sol en el movimiento planetario mediante la atracción magnética (cf. Hacyan, 2004, p. 62). De estas investigaciones, Maxwell fijará el concepto de "campo", como el "conjunto total de líneas de fuerza" (Einstein e Infeld, 1938/1961, pp. 112 ss.), lo que implicará un estudio de la realidad según otras leyes físicas. El resultado de estos hechos es una reformulación total de la Naturaleza, lo cual implica, consecuentemente, una reformulación del concepto de tiempo, y es que este ha dejado de ser útil para la descripción de los movimientos mecánicos tal como los postuló la física newtoneana.

En este contexto, la posición de Einstein será radical. De lo que se trata para la física es ahora de buscar un parámetro de medida en vistas a la descripción de esta "nueva" Naturaleza, aquella que ya no es material, sino más bien electromagnética. En lugar del tiempo, reducido al sujeto y considerado como variable respecto de la velocidad del sistema de referencia en el cual se encuentre el observador, habrá que fijar otro criterio de orden. Alguno que se halle en la misma realidad, pero que sea inalterable. Este será el rol de la velocidad de la luz. De esta manera, ya no habrá que depender de un criterio "subjetivo" y, por ende, impreciso e irreal, como parece ser el tiempo, para continuar con el estudio del movimiento en la realidad observada. Ella misma, podríamos decir, ofrece un parámetro de medida efectivo, confiable y útil para determinar qué es lo que 
ocurre en un "antes" y un "después" (cf. García Doncel, 1989, p. 57). La teoría de la relatividad, en definitiva, parece ser una teoría ocupada en corregir esta "problemática" relatividad del tiempo. ${ }^{2}$

Así es como quisiéramos destacar algunos aspectos importantes que surgen de estas discusiones. Se ha visto que el problema del tiempo aparece en la física como necesario en función de su intento de explicar y describir el movimiento. Esto quiere decir que las modificaciones que ha experimentado su definición se circunscriben a problemáticas que acaecen desde la confrontación con un fenómeno que no es propiamente él mismo. Esto implica, en definitiva, que la física no se ha confrontado con el tiempo en estricto rigor. Ella, más bien, ha estado reelaborando una herramienta en vistas a otros fines (cf. Elias, 1989/2013, p. 121). El sentido de sus reformulaciones del concepto de tiempo viene a traducirse, entonces, en el esfuerzo por avanzar en su trato con lo efectivamente observado. Allí donde el tiempo no sirve más para sus pretensiones, será donde este experimentará nuevas definiciones. El tiempo no parece ser otra cosa que una herramienta más de la física para ocuparse del mundo. El tiempo, más allá de ser su propio tema, le es, por tanto, funcional.

El problema que advertimos acá es que el concepto actual de tiempo nada nos puede decir del propio fenómeno del tiempo, ya que desde un principio este ha sido entendido en el horizonte de una ocupación con el mundo y desde ella se le ha definido. Formular al tiempo como parámetro de medida (cf. Heidegger, $1987 / 1994$, p. 73) implica ya errar en la pregunta que ha de plantearse por él mismo. De esta forma, entendemos que la relación con el tiempo por parte de la física es interesada. ${ }^{3}$ Dicho interés, podríamos decir, ya es parte de un tipo de relación particular con el mundo: el ocuparse fáctico con el mismo. Obtenemos así que el sentido de la física es el del ocuparse. En efecto, dicho ocuparse se muestra como el horizonte primero de las pretensiones que subyacen a su esfuerzo explícito por reelaborar el concepto de tiempo. Por lo cual, comprendemos que esta relación ocupada con el mundo determina la vigencia de sus definiciones del tiempo. Pues bien, quisiéramos fijar este carácter ocupado de la física como horizonte de comprensión del tiempo que ella utiliza, en cuanto será en concreto dicha ocupación aquel fenómeno que deberemos investigar para comprender cuáles son sus propios fundamentos. En última instancia, será en este contexto donde podremos caracterizar a la ciencia respecto del sentido propio de su ejercicio.

\section{EL TIEMPO DESDE LA OCUPACIÓN CON EL MUN- DO Y EL TIEMPO DE LA FÍSICA}

Las discusiones anteriores señalan que el tiempo en la física, independientemente de sus reformulaciones, viene a ser el parámetro de medida del cambio y movimiento de la Naturaleza. $Y$ es que este ha estado al servicio de una explicación adecuada de la realidad misma. Con ello, se ha destacado que su definición clásica parece motivarse desde una particular ocupación con la realidad. En lo que sigue, entonces, deberemos detenernos en dicha ocupación para comprender cómo es que esta, que no es otra que la del científico mismo, parece fundar el concepto de tiempo propio de la investigación científica.

En todo caso, esta reconducción del concepto de tiempo al científico no pretende decidir si el tiempo es o no subjetivo. Más bien, afirmamos que, indiferentemente de cómo fácticamente se le haya entendido, i.e., ya sea como una entidad cuyo flujo es radicalmente autónomo tanto respecto del mundo natural como del científico, o ya sea como un constructo abstracto, resultado de las sensaciones del observador, ambas nociones emergen desde una ocupación primaria que se concentra en hallar un parámetro de medida del cambio y movimiento de la Naturaleza. Ahora bien, desarrollar esta reconducción a la ocupación en tanto que relación con el mundo por parte del científico, atiende a buscar un suelo primario desde donde acceder al fenómeno del tiempo en la física. Es acá donde el pensamiento de Heidegger puede guiarnos. Las siguientes consideraciones suscribirán el carácter existencial de sus propios planteamientos. ${ }^{4}$

El mentado carácter existencial que destacamos de Heidegger implica reconducir al ente a su pertenencia con el existir, de modo que la conceptualización que sea utilizada en dicha tarea indique al ente desplegándose en una relación con el sí mismo. Este es precisamente el modo de proceder del planteamiento heideggeriano del tiempo. Luego, no es un gesto arbitrario acceder al mismo desde la lectura del reloj, como lo hace Heidegger, cuando precisamente en dicha "lectura" se plantea metódicamente el problema del tiempo en el contexto de quien lo "lee". En concreto, los análisis comenzarán desde lo que podría formularse como un "contar con el tiempo" (Rechnen mit der Zeit) (cf. Heidegger, 1987/1994, p. 77; 1975/1997, pp. 364 ss.; 1927/2001, p. 416), en el momento en que consultamos la hora en un reloj.

Pues bien, cuando contamos con él, es decir, cuando tenemos tiempo o no disponemos del mismo, nuestra relación no es primariamente con este, sino con 
aquello con lo cual nos ocupamos durante un lapso temporal determinado. Tenemos o no tenemos tiempo, por tanto, cuando nos está permitido ocuparnos de aquello que debemos o cuando esto no es posible, puesto que nos hallamos absortos en otras ocupaciones. Acá se muestra un primer rasgo del tiempo en relación a nosotros, a saber, un "para algo" que le constituye, y que Heidegger denominará significatividad (Bedeutsamkeit) (cf. Heidegger, 1975/1997, p. 370; 1927/2001, p. 414). ${ }^{5}$ El tiempo remite, "indica hacia un para algo" (Heidegger, 1987/1994, p. 58): hacia un ámbito de ocupaciones determinadas.

Desde tal significatividad del tiempo aparecerá un segundo carácter del mismo en el contexto del existir. Cuando decimos: "ahora me ocupo de tal o cual asunto", el tiempo muestra una cierta puntualidad que no tiene sentido sino desde la ocupación misma. La expresión "ahora, cuando ocurre esto o aquello" refiere, en consecuencia, a una situación constituida primariamente por un ocuparse definido y concreto en la que nos hallamos. Desde lo anterior es que podemos entender que el "antes", el "ahora" y el "después" que podemos delimitar en términos temporales se constituyen por determinadas ocupaciones dadas en un ahora específico, ya sea en un "ahora" pasado, presente o futuro. Este segundo carácter es el que Heidegger denominará "databilidad" (Datierbarkeit) (1975/1997, pp. 370 ss.). Dichos "ahora", no obstante, poseen un determinado "intervalo" (Spanne), una extensión propia (Erstrecktheit), que correspondería a una tercera característica de este tiempo vivido. Así, "este invierno me dedicaré a tal o cual asunto" es una expresión que mienta implícitamente un "ahora...hasta” (bis dahin) (Heidegger, 1975/1997, pp. 372 ss.). Los "ahora", podríamos decir, que delimitan un ocuparse concreto, no se identifican con el tiempo de un cronómetro. Un "ahora" puede durar meses o años, como cuando decimos: "entonces, cuando era niño...", señalando un periodo temporal que ciertamente se prolongaría por muchos años "cronométricos". Por último, estos "ahora", constituidos por el ocuparse, son accesibles para todos, puesto que cuando se dice "ahora que escribo este trabajo" o "mañana, cuando comience con las lecciones", se trata de expresiones que todos aquellos que las escuchen podrán comprender. En otras palabras, este tiempo vivido posee, a su vez, un carácter público (Öffentlichkeit), i.e., compartible por el otro, dado a que las ocupaciones referidas son para todos manifiestas (cf. Johnson, 2012b).

Entonces bien, el hecho de que en estas consideraciones Heidegger destaque la identidad entre el modo de ser del tiempo y del mundo es un asunto que debe ser considerado. En efecto, la significatividad que le correspondería al tiempo es también aqueIla que describe el mundo propio. En este sentido es que Heidegger identificará este tiempo con el tiempo del mundo (Weltzeit) (cf. Heidegger, 1975/1997, p. $370 ; 1927 / 2001$, p. 414). Tiempo y mundo, podríamos decir, se vinculan en estas consideraciones en cuanto son instancias de un fenómeno primario como es, ante todo, el del ocuparse (Besorgen). Así, del carácter "para algo" del tiempo mundano se rinde cuenta de la co-presencia del tiempo, según un "antes y un después", pues, esos "ahora" se constituyen fundamentalmente en una relación existencial determinada: la de la ocupación con los entes del mundo.

Fundamental para estas discusiones es advertir que, si bien, el ocuparse del existir se ha mostrado como aquel que, en efecto, llevaría a la delimitación del tiempo mundano, decíamos también que las ciencias son, ante nada, un tipo de ocupación con el mundo. No obstante, se advierte que el tiempo con el que tratan las ciencias no parece ser el mismo que aquel descrito en las caracterizaciones heideggerianas. El tiempo clásico de la física se presenta más bien como un flujo progresivo, adyacente a los fenómenos físicos. En el caso del traslado, el tiempo es entendido en relación con el cambio de lugar que presenta un móvil. Podría decirse: "antes, el móvil se encontraba en el punto A, ahora, en el B, y después, en C". Dichos "antes", "ahora" y "después", se traducen para la física en números. En el segundo "2", el móvil estaba allá, en el segundo " 4 ", se encuentra acá, etc. Sin embargo, la consecución de segundos transcurre "retraída en sí", sin incidir en el móvil mismo. En otras palabras, los "ahora" del movimiento, expresados en segundos, carecen de todo vínculo con lo que ocurra en ellos. Se trata de un tiempo entendido como una "consecución de ahora" indiferente a los eventos contenidos por este (cf. Heidegger, 1987/1994, p. 55). La autonomía del tiempo que detectábamos en Aristóteles, parece adquirir ahora el sentido concreto de "indiferencia" respecto a los eventos de la realidad.

Así es como se puede observar que la física parece haber modificado su experiencia del tiempo respecto de aquella del vivir cotidiano. Como se observa, a la experiencia del tiempo de la física le pertenece una anulación del carácter "para algo" del tiempo cotidiano, es decir, en ella se ha suprimido su significatividad. Dicha supresión no es baladí. Ella es la condición de posibilidad de que otro carácter del tiempo también se anule: la databilidad. En efecto, en cuanto 
la co-experiencia del "antes", "ahora" y "después" se desliga de aquello con lo que el existir está en aquellos respectos ocupado, el tiempo, como es el caso del calendario, se muestra como un conjunto de lapsos vacíos y dispuestos a ser "rellenados" por algún hecho que eventualmente pueda acaecer. Esto dará lugar a que la extensión del tiempo, el carácter dimensional que destacaba Heidegger de Aristóteles, que ciertamente se conserva en el tiempo de la física, haya perdido todo suelo, y el tiempo se experimente como una instancia desligada completamente de lo que se observa, es decir, adyacente (cf. Heidegger, 1975/1997, p. 371; 1927/2001, p. 422). El tiempo de la física se muestra como un fenómeno en el cual ni los hechos ni el observador pueden incidir, y que es igual para todos, como algo "meramente presente". Aparece, entonces, una cierta "objetividad del tiempo", posibilitada por la anulación de su significatividad. En definitiva, el tiempo, desarraigado de la ocupación fáctica, se presenta como aquel continuum constituido por el pasado, el presente y el futuro en el sentido de un mero escenario pasivo que contiene eventuales hechos (cf. Heidegger, 1975/1997, p. 385).

Como se puede ver, la definición del tiempo nos ha conducido a comprender que su articulación parece emerger de una determinada relación del científico mismo. Ha sido la detección de su ocupación la que nos lleva a entender cómo es que el tiempo cotidiano se modifica hasta la obtención de un tiempo indiferente a los hechos que en él ocurran. Una caracterización inicial de esta ocupación señala que la vivencia primaria del tiempo en tanto "para aquello en lo que nos ocuparemos" anula precisamente la relación "para", propia del existir, y lo que adquiere primacía efectiva es el "algo" de la ocupación, es decir, el mero objeto de estudio. En el caso de la física, este parece ser el movimiento y el cambio, y el tiempo, al interior de su investigación, es comprendido como un mero escenario de los eventos estudiados. La caracterización aristotélica del tiempo en cuanto número, en cuanto adyacente, se radicaliza como un simple trasfondo vacío e indiferente a lo que lo rellene.

De esta manera, las discusiones nos llevan a la necesidad de indagar qué tipo de ocupación con el mundo es aquella que se caracteriza por un olvido de su íntimo sentido, de su "para", para privilegiar aquello con lo cual ella se ocupa. Como se puede advertir, con dicha tarea ya nos aproximamos a lo que nos proponíamos en este trabajo, i.e., caracterizar el sentido propio del quehacer científico mediante su relación con el tiempo. Como veremos, dicho modo de rela- ción es el que Heidegger denominará caída (VerfaIlen). Pues bien, a continuación deberemos entender cómo es que la condición caída de la ciencia posibilita su propia noción de tiempo y, con ello, cómo es que ella devela lo que ella misma propiamente es en su auténtico ejercicio.

\section{EL QUEHACER CIENTÍFICO DESDE LA CAÍDA Y EL PRIVILEGIO DEL AHORA}

Por lo pronto, podemos decir que la ciencia es un tipo de ocupación con su mundo, un modo de relación existencial en el cual todo redunda en el objeto de su ocupación. La tarea será, por consiguiente, indagar en el sentido preciso de esta ocupación. Para ello, las siguientes discusiones deberán retomar la lectura del reloj anteriormente referida, sin embargo, en un sentido distinto al ya comentado. No se trata, en lo que sigue, de entender la lectura del tiempo en este artefacto en cuanto un "contar con el tiempo". Más bien debemos fijarnos ahora en el desplazamiento de sus manecillas.

Según lo expone Heidegger, cada avance del segundero indicaría implícitamente un "ahora aquí", "luego aquí", etc. No obstante, mediante su paso advertimos que el "ahora" pasado que percibíamos ya no es, mientras que el que existe es el ahora "apuntado", el mismo que dejará de ser en la medida en que el segundero avance al segundo siguiente. La lectura del reloj nos pone ante un fenómeno decisivo para nuestras discusiones. Se trata de una aporía que ya San Agustín planteara: "Pero aquellos dos tiempos, pretérito y futuro, ¿cómo pueden ser, si el pretérito ya no es él y el futuro todavía no es?" (398/1974, p. 479). En la cita se aprecia un rasgo particular del tiempo. En efecto, su flujo parece darse en la forma de un movimiento de lo que "ya no es", a lo que "efectivamente es", para luego pasar a lo que "aún no es". La pregunta, dentro del contexto de nuestras discusiones será, entonces: ¿qué es lo que posibilita esta experiencia del tiempo como un tránsito desde el ser al no-ser?

Es en este contexto cuando las consideraciones heideggerianas señalan una primacía del presente que quisiéramos revisar. Lo pasado, según se plantea, sería lo que "ahora ya no es" (Jetzt-nicht-mehr), mientras que el futuro, lo que "ahora no es aún" (Jetztnoch-nicht) (cf. Heidegger, 1975/1997, p. 367). En otras palabras, en la lectura del reloj se encuentra implícito un "ahora" que decide entre lo que "ya pasó" y lo que "vendrá". Un ahora que parece ser el referente según el cual se decide entre pasado y futuro, y que se extenderá "hacia atrás" y "hacia adelante" en el 
sentido de un no-ser. Atendamos, por lo pronto, al movimiento que se ha efectuado en estas consideraciones. El caso es que la noción de tiempo de la física asume un avance desde el pasado al presente, y luego, al futuro. Lo que hace Heidegger, por su parte, es aclarar este trascurso lineal haciendo énfasis en el presente efectivo, justo cuando consultamos el reloj, y mediante él determinar para "atrás" al pasado como lo que fue, así como hacia "delante", al futuro, como lo que será. Dicho de otra manera, a la determinación del tiempo como consecución de "ahora" subyace una relación del existir que ya está instalada en dicho presente efectivo. El tiempo de la física y de las ciencias en general se enraizaría, entonces, existencialmente en un privilegio del ahora desde el cual pasado y futuro son advertidos. Dicho privilegio, empero, no es un rasgo del tiempo mismo en juego. Este viene a caracterizar más bien la relación con dicho tiempo. Se trata de una relación que fundamentalmente radica en privilegiar al objeto de su ocupación $y$, a la vez, en conceder primacía al modo de tiempo que conocemos como "presente". Ambos caracteres ya comienzan a indicar la manera como el quehacer científico se despliega. Anteriormente decíamos que se trata de aquel modo del existir que Heidegger denominara "caída". Por tanto, deberemos aclararnos en sus caracteres fundamentales.

El existir cotidiano no implica solo una relación con el mundo en la cual se da un mero "hallarse" unívocamente en relación hacia las cosas. Un momento importante de dicha relación es que los entes mismos del mundo apremian, por su parte, al existir, de modo que este se deja "arrastrar" por ellos (cf. Heidegger, 1976/1995, p. 213). En términos heideggerianos, este momento de la relación con el mundo se expresa formalmente en la estructura del cuidado (Sorge) como un "estar ya junto a..." (schon Sein-bei...) que implica, a su vez, un estar absorto con el ente "junto al cual" nos hallamos (cf. Heidegger, 1976/1995, pp. 241 ss.; 1927/2001, p. 61). Es importante tener en cuenta que dicho "ya estar junto a..." no es una constatación de un hecho fáctico, como si expresara que un sujeto se hallaría "meramente próximo a otras entidades". Este caracterizará la relación misma con el ente. Es decir, en la cotidianidad, a la experiencia del mundo le pertenece el sentido de encontrarse "ya entre" las cosas, y dicha relación se ejecuta como una tendencia a privilegiar al ente con el cual el existir se ocupa.

Precisamente, este movimiento general de la caída es el que queremos identificar como un rasgo decisivo de la física y del quehacer científico mismo respecto del tiempo y de su mundo. Desde las consideraciones heideggerianas se advierte que el existir caído presenta una "avidez de lo nuevo", es decir, se constituye en un modo de mirar su mundo mediante el cual, al tener privilegio el objeto del ocuparse, rápidamente se pasa de un objeto de la ocupación a otro. Se trata de la curiosidad (Neu-gier). Esta tendencia implica un afán por abandonar rápidamente el objeto de la ocupación actual, lo cual da lugar a un característico "desarraigamiento" (Aufenthaltslosigkeit) que deja al existir caído sin un lugar propio de permanencia. Así, la caída viene a entenderse como una relación del existir en la que a este, arrastrado por el mundo, le es dado "dispersarse" en cada objeto nuevo de la ocupación con el que pueda tratar (Zerstreuung) (cf. Heidegger, $1927 / 2001$, p. 347), en tanto todo nuevo objeto de la ocupación es, al igual que otros, digno sin más de ser procurado (cf. Johnson, 2013). En su condición caída y ocupada, podemos decir en definitiva, el ejercicio científico parece vivir para las cosas eminentemente presentes, es decir, aquellas que pueden ser objeto efectivo de su ocupación. Así llegamos, entonces, a la temporalidad en la cual el quehacer científico acaece, esto es: el presente. Al respecto, empero, hay algunos aspectos que aclarar.

\section{LA CIENCIA Y SU PRESENTE}

Hasta este punto, se ha discutido que la noción del tiempo de la física no nace desde una preocupación por el tiempo en cuanto tal, sino por el movimiento y el cambio del mundo natural. Dicha observación nos condujo a observar que la física se constituye en una ocupación con su entorno que privilegia el objeto de su ocupación y, podemos decir ahora, en tanto que caída en este objeto, esta articula al tiempo como una herramienta funcional a sus propias pretensiones. El fundamento de la ocupación científica en general es, por tanto, aquello con lo que actualmente se ocupa. Con ello entendemos que, en su tendencia caída, la ciencia se ejecute como una posibilidad del existir cuyo horizonte temporal sea ante todo lo presente. En aquello que se presenta, en vistas al objeto actual del trato, la ciencia comprende al mundo en general y sus propias tareas. Es por ello que el presente se muestra como el horizonte temporal del quehacer científico, ya que lo actual es lo auténticamente válido, lo único "existente". Dicho modo de relación primariamente presente con el mundo le conduce, a la vez, a entender que aquello con lo cual ella se ha ocupado ya no sea, y aquello con lo cual ella se ocupará, aún no sea, ya que ambos no son objetos del trato efectivo. Por tanto, la actualidad del presente, 
como aquel "cuando" en el que el científico se ocupa de facto, es el criterio último que funda su noción del tiempo como un transcurso de ahoras, donde los pasados y los futuros no son.

De esta manera, el modo del existir denominado caída comienza a rendir cuenta de algunos rasgos característicos del ejercicio científico. En primera instancia, comprendemos que la ciencia se despliegue privilegiando el dato presente percibido. El privilegio del hecho observado, tan proclamado por la ciencia experimental, parece arraigarse en el privilegio del presente como su auténtica temporalidad, en tanto que este es el horizonte de su relación ocupada y caída. En efecto, será lo que ahora es objeto de la ocupación, a saber, el momento cuando el ente se presenta auténticamente, lo que tiene validez. En este contexto existencial, se presenta la decisividad de la empiria como campo auténtico de su estudio. Asimismo, en la "tentación" de lo dado en el presente, la ciencia puede comprender también "lo pasado". Es la carencia de actualidad del pasado la que ha decidido que este ya no sea. No obstante, su "irrealidad" no puede considerarse una pura nada. Dado a que la ciencia habita en la pretensión de ocuparse con lo actual y se esfuerza por optimizar dicho trato, ella ha asumido que el movimiento y cambio presente no sería espontáneo, sino el resultado de eventos precedentes. De esta manera, el quehacer científico se dedica a "actualizar", es decir, a presenciar nuevamente lo pasado, esto es, se preocupa por volverlo al "ser" en función de determinar qué configuración de eventos ha dado paso al hecho actual de su ocupación. Este esfuerzo se traducirá en leyes que se expresarán de la manera: "Si A, entonces, B", donde A será la causa, es decir, el factor o el conjunto de factores que se derivará en $\mathrm{B}$, esto es, el efecto, es decir, lo que es objeto auténtico de estudio. Por otra parte, el no-ser propio del futuro se distinguirá de aquel que caracteriza al pasado, puesto que, en tanto que "aún no es", será objeto de la ocupación. En todo caso, nuevamente es el privilegio de la ocupación actual la que determina su no ser, aunque ahora se trata de preparar un trato fáctico eficiente con el evento esperado. Este será el sentido de la predicción. ${ }^{6}$ Esta viene a ejecutarse en el sentido de preparar las condiciones adecuadas para que cuando el evento se presente, la ocupación con él sea la óptima. No obstante, a pesar de que la predicción se relacione con el futuro, todo lo que se hace en función de este, acaece auténticamente en el presente. La ciencia, podemos decir, vive anclada en lo presencial. Así es como en esta relación de carácter existencial, en la caída, el pasado y el futuro se su- bordinan a la actualidad de la ocupación. Ante nada, el pasado y el futuro son experienciados por el trato científico como instancias que, "no siendo", cada una a su modo, son dignas de atención solo al servicio de la presencialidad efectiva. ${ }^{7}$

Desde la primacía del presente característica de la relación caída de la ciencia, es posible, en última instancia, comprender la noción de tiempo como aquella secuencia de avance del pasado al presente $y$, luego, al futuro, comprendiendo el sentido del no-ser que a cada instancia que no sea la presencial le pertenece. Sin embargo, resta entender ahora la comprensión del tiempo como instancia objetiva, mediante la cual se develarán los últimos caracteres del quehacer científico que aclararán su íntimo sentido. Los caracteres descritos de la caída no agotan plenamente el hecho de que el tiempo sea comprendido como una instancia desligada del existir ocupado. En lo que sigue, debemos entender cómo es que este existir caído en su mundo puede experimentar una posterior modificación.

Por lo pronto, la caída es una posibilidad del propio existir cotidiano mediante la cual este, antes de entrar en un trato con "cosas", se relaciona con el ente intramundano en tanto que "útil" (Zeug) (Heidegger, 1927/2001, p. 68). Desde las consideraciones heideggerianas se advierte que dicho útil expresa el nivel primario de comparecencia del ente del mundo según el modo de ser de "lo a la mano" (Zuhandenheit) (Heidegger, 1927/2001, p. 83). En dicho nivel, al existir le es dado caer en la significación del ente, haciéndose cargo del mismo en cuanto un "para algo". Esto es lo que permitirá entender la inmediatez del ente que le comparece al existir como perteneciente a un "todo remitivo" (Bewandtnisganzheit) (Heidegger, 1927/2001, p. 84), cuyas remisiones radican en su carácter de "para algo". Lo importante para nuestras discusiones es que el "lugar" (Platz) (Heidegger, $1927 / 2001$, p. 102) que le corresponde al ente inmediato en dicho "todo remitivo" está muy lejos de identificarse con el espacio físico que él "rellenaría" entre otras "cosas". Su "lugar" es el que se da desde su propio "para qué", por tanto, lo que el ente con el carácter de lo "a la mano" sea, acaece dentro del todo remitivo que caracteriza la inmediatez del mundo circundante (Umwelt) para el existir ocupado. En este contexto, el existir cae, se deja arrastrar por los "para algo" que descubre en su propia ocupación, aquella que, como sabemos, remitirá, en última instancia, a un por mor de "sí mismo" (Worumwillen), es decir, al mismo existir (cf. Heidegger, 1927/2001, p. 87). 
Pues bien, es posible que acaezca una modificación teórica de esta experiencia del mundo circundante. Esta, sin embargo, no consistiría precisamente en la pérdida total de la praxis (cf. Heidegger, 1927/2001, p. 357). De hecho, el científico de todos modos tiene un trato práctico con aquello que encuentra en su proximidad al realizar su investigación. Este manipula microscopios, prepara muestras, etc. Sin embargo, la manera como el quehacer teórico se instala en su mundo corresponde a un modo característico y diferente al inmediato. El quehacer teórico, señala Heidegger, tiene sus "propias maneras de permanencia" (1927/2001, p. 358) en su mundo. Atendiendo al caso que el mismo Heidegger expone, podemos decir que en una relación cotidiana, el martillo poseería su "lugar" respecto de su "martillar para", sin embargo, afirmar "el martillo es pesado" muestra ya la modificación en juego. Lo decisivo en este caso no es que no se experimente más el carácter de útil del martillo, sino, ante nada, que acaezca una pérdida de su propio "lugar" en aquel contexto remitivo con el cual el existir se hallaba en alguna relación (cf. Heidegger, 1927/2001, p. 361). El ente es arrebatado de su lugar en las remisiones constituidas por los "para algo". El lugar existencial del útil pasa a ser el espacio indiferente, sin vínculo existencial (cf. Heidegger, 1927/2001, p. 362). Esta ruptura entre el existir y su mundo viene a darse, por tanto, como un olvido del contexto desde el cual comparece el ente inmediato. Es entonces cuando este se experiencia según el modo de ser que Heidegger denominará "la mera presencia espacial" (Vorhandenheit) (cf. Heidegger, 1927/2001, p. 96).

Así, esta modificación que deriva al existir teórico nos permite regresar nuevamente a la física. Si ya discutíamos que el objeto de su ocupación es el movimiento y el cambio en la Naturaleza, ahora vemos que dichos fenómenos, en la tendencia desligadora de la actitud teórica, pasan a ser vividos como instancias cuyo único puesto de ubicación es el espacio geométrico, es decir, se comprenden como instancias completamente indiferentes de la propia ocupación y, por ende, se vivencian como radicalmente desvinculados del observador que con ellos se ocupa. En términos temporales, podríamos decir que la física, "apresada" por la presencialidad de la Naturaleza, "olvida" que esta, tal como se le presenta, deviene en el horizonte de su propia ocupación. El ocuparse mismo, el privilegio de lo presencial inherente a él y su correspondiente tendencia por procurarse lo que se presenta solo en el trato efectivo, implican una definitiva anulación de la pertenencia de quien se ocupa, el científico mismo, y de sus propias pretensiones. La ocupación misma y su sentido han de quedar, pues, ocultas ante el protagonismo del objeto estudiado. El mundo es vivido como "algo para sí mismo", en el anonimato de quien procura de él. En esta pérdida del sentido existencial, la realidad pasa a ser entendida, en palabras de Heidegger, como "lo que se encuentra constantemente presente" (ständig Vorhandenes) (1927/2001, p. 362). Resulta así la noción de "materia" y el privilegio temático de la misma, ciertamente como instancia "retraída en sí" y "a la espera de ser observada". Asimismo, la noción de tiempo lineal responde y es el resultado de esta modificación existencial. Dentro de esta, lo que importa, entonces, es fijar parámetros autónomos, como herramientas para el estudio de fenómenos igualmente autónomos. Así es como acaece una experiencia del tiempo en tanto que flujo progresivo de diversos "ahora", cada cual, por cierto, comprendido en una desvinculación radical respecto del existir que se ocupa de los mismos. El tiempo de las ciencias, en consecuencia, pasa a ser comprendido en su objetividad, es decir, en una indiferencia existencial del mismo ejercicio vital del cual este emerge. ${ }^{8}$

\section{CONSIDERACIONES FINALES}

Pues bien, el presente trabajo ha intentado delimitar el sentido fundamental del ejercicio científico a partir del modo de trato con el problema del tiempo. Siguiendo la lectura heideggeriana de Aristóteles, se ha visto cómo es que su caracterización en tanto que "número del movimiento" resulta en una concepción del tiempo mismo como una instancia autónoma respecto tanto del móvil como del movimiento mismo, entendiéndolo como un continuo que contendría hechos acaecidos en su interior, mientras este permanecería, por así decir, sin ser afectado. Dicha autonomía es la que habría sido recepcionada justamente en la investigación científica de la realidad. En el caso particular de la física, se advirtió, en efecto, que tal noción de tiempo fue, en un principio, de suma utilidad para que esta pudiera ocuparse de su objeto auténtico de estudio: el movimiento y el cambio en la Naturaleza. Mas, en la física no parece hallarse una confrontación misma con el fenómeno en cuestión, sino que la vigencia de la noción de tiempo en ella parecía depender de la utilidad que esta proporcionase a la investigación de la realidad contingente. En otras palabras, el caso de la física muestra cómo es que el ejercicio científico opta por alguna definición de sus conceptos fundamentales en vistas a su funcionalidad, entendida esta como el criterio último de decisión para asumir o descartar conceptos en su investigación. La ciencia, se dijo en este contexto, radicaría, en consecuencia, en un modo de trato interesado con el mundo, y dicho interés 
no puede ser entendido sino como la expresión de una relación esencialmente ocupada que constituiría el íntimo sentido del ejercicio de la misma ciencia.

Tal ocupación, por lo demás, aparecía en estas consideraciones como el ámbito en el cual la vivencia cotidiana del tiempo, según Heidegger la entiende, parecía ser desvinculada del propio existir. Así, la pregunta por qué tipo de ocupación es aquella que, atendiendo a las cosas de su mundo implica también una desvinculación del mismo, fue respondida caracterizando tal relación ocupada como una, en términos heideggerianos, caída y teórica. Esto es, el ejercicio científico fue entendido como un tipo de ocupación que, arrastrada por el objeto de su trato, termina, a su vez, por olvidar cualquier tipo de relación íntima con él. En otros términos, el quehacer científico se presentó, desde estas consideraciones heideggerianas, como uno que, caído en la presencia de las cosas entre las cuales se haya perdido, entiende que la efectividad de la realidad no es otra que lo que únicamente está ahí, delante de él, dispuesto para su trato. Tal es la relación existencial fundamental que nos llevó a pensar que la noción de tiempo lineal, en tanto que progreso del pasado al presente y, luego, al futuro, no es otra que el resultado de la exigencia por tratar y optimizar el trato presente. Tal condición caída y su respectiva tendencia teórica que desvincula de sí lo que aparece se destacó, así, como el contexto existencial en el cual la definición de tiempo se delimitaba como una entidad autónoma y objetiva, es decir, experimentable por todos de la misma manera. Por esta vía es que las presentes consideraciones intentaron destacar que la noción de tiempo científico es el resultado de una relación existencial constituida desde el privilegio del presente.

Pues bien, el énfasis en la tendencia caída y teórica ha sido decisivo para una caracterización de la ciencia. Esta se ha mostrado, en efecto, como el origen existencial, no solo de aquellos conceptos que guían su propio ejercicio, sino, ante nada, del "mundo" mismo que pasa a ser objeto de su consideración. Así, este presente que se devela en el seno de su quehacer se exhibe como una dimensión temporal prima- ria y privilegiada en la cual la ciencia permanece en esencia anclada. Finalmente se advierte que con la caracterización del ocuparse científico ya estamos en condiciones de comprender la íntima naturaleza de la investigación científica. La ciencia, podemos decir, es, en su esencia, una particular posibilidad del existir de carácter eminentemente presencial y es dicha "presencialidad" el fundamento del ser de aquel ámbito de acción en el cual a la ciencia misma le es dado caer: su "realidad".

No obstante, estas consideraciones existenciales no han pretendido sino solo esbozar una caracterización vital del problema de la ciencia mediante el pensamiento de Heidegger. El problema tratado, empero, no se agota acá, sino más bien se abre a posteriores reflexiones. Reflexiones que, por cierto, han de ejercerse como una investigación atenta a la relación entre el tiempo del mundo y lo que Heidegger entenderá como la temporalidad originaria del Dasein: el futuro (cf. Heidegger, 1927/2001, p. 365). ${ }^{9}$ Por lo pronto, este estudio sobre el quehacer científico se constituye en un esfuerzo por aclarar una posibilidad del existir indagando precisamente en el sentido temporal que la funda, de manera que aquel concepto existencial de ciencia que el mismo Heidegger consideraría como necesario para comprender su esencia (cf. Heidegger, $1927 / 2001$, p. 357) pudiese ser pensado hasta el nivel que este mismo consideró como el originario en el ámbito de lo humano, esto es: el fenómeno del tiempo. Desde tal perspectiva, a nuestro juicio, es que la ciencia en tanto tal puede abrirse a la posibilidad de confrontarse con sus conceptos fundamentales atendiendo a la relación vital que ella misma es en tanto modo de ser del Hombre. ${ }^{10}$

\section{AGRADECIMIENTOS}

Este trabajo se realizó con el apoyo del Programa Mece 2 Educación Superior, Proyecto Fro0901: “Fortalecimiento de las Ciencias Sociales y Humanidades en la Universidad de La Frontera" del Ministerio de Educación, y del Proyecto DIUFRO-DI 14-005: "La presentación futura del Ahí".

\section{NOTAS}

1. La pertinencia de plantear una revisión existencial del ejercicio científico mediante el modo como la física ha tratado con el problema del tiempo a partir del pensamiento de Heidegger se comprende a partir de dos razones. Por una parte, el interés por la ciencia como posibilidad del existir es un aspecto re- currente en el pensamiento heideggeriano como modo de aclarar cómo esta se distingue del filosofar (cf. Johnson, 2012a). Empero, la confrontación de Heidegger con ella no permanece solo en la descripción existencial del modo teórico de trato con el mundo. Heidegger demostró reiteradamente conocer

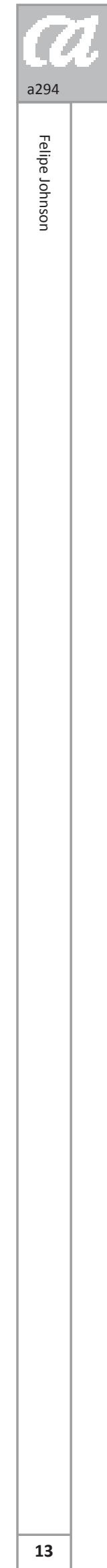

los avances de la investigación científica particular, como ocurre con la física. Así lo atestiguan referencias a Einstein (cf. Heidegger, 2001, p. 9; 1994, pp. 73 ss.), pero también a Heisenberg. Respecto de este último, la discusión gira en torno al pensar calculador que lleva a la física a considerar a la Naturaleza solo 
en cuanto fuente de recursos (cf. Heidegger, 2000, p. 24). Asimismo, Heidegger refiere a la física moderna como fundada en los rasgos esenciales de la técnica y su tendencia al dominio de la Naturaleza, señalando a Galileo, Heisenberg y Max Planck como concreciones de este pretendido señorío del mundo (cf. Heidegger, 2005, p. 355). Para la relación entre Heidegger y Heisenberg (cf. Pöggeler, 1993). Cabe señalar, sin embargo, que el presente estudio no se inscribe en estas tesis propias del período tardío del pensar heideggeriano.

2. Heidegger comentará que la Teoría de la Relatividad se movería en la tendencia de "resguardar la invariabilidad de las leyes del movimiento" (2001, p. 10).

3. Destacamos el concepto husserliano de "interés" (Husserl, 1992, p. 143) como una caracterización del ejercicio científico que lleva a comprender el "olvido de sí mismo" inherente a su despliegue (cf. Johnson, 2011).

4. Es importante aclarar que las siguientes consideraciones se enmarcan en el pensar del tiempo del Heidegger de la época de Ser y tiempo, es decir, atenderá al problema de la fundación del tiempo cronológico en la experiencia de tiempo cotidiano. No obstante, la problemática del tiempo se extiende más allá de este periodo. Esta será tratada por Heidegger, en efecto, en el contexto de su crítica a la técnica, con los énfasis que este daría, en esta fase intelectual tardía, a la tendencia propia de la ciencia moderna de afianzar su señorío calculador en la

\section{BIBLIOGRAFÍA}

Agustín de Hipona. (398/1974). Las Confesiones. Madrid: BAC.

Aristóteles. (IV a. C./1956). Physica. Londres: Oxonii e typographeo clarendoniano.

Aristóteles. (IV a. C./1957). Metaphysica. Londres: Oxonii e typographeo clarendoniano.

Aubenque, P. (1962). Le problème de l' être chez Aristote. Paris: PUF.

Batovanja, V. (2008). Heidegger und die Wissenschaft. Synthesis Philosophica, 46, pp. 401-411.

Brentano, F. (1862/1960).Von der mannigfachen Bedeutung des Seienden nach Atistoteles. Darmstadt: Wissenschaftliche Buchgesellschaft.
Naturaleza. Para una descripción de las etapas del pensamiento heideggeriano respecto al problema del tiempo cf. González (2008, pp. 23-27).

5. Más tarde, Heidegger (1994, p. 58) precisará al tiempo como "deutsam", i.e., "indicador", y no "be-deutsam", ya que, en cuanto "significativo", resuena la idea de "simbolización", inadecuada a su carácter "referencial".

6. Ya se observa la importancia de la predicción en la investigación científica desde la pretensión última del "espíritu positivo". Se trata de "ver para prever" (Comte, 1898, p. 23), i.e, de intervenir en los hechos para modificar su cumplimiento. En este sentido, Heidegger reconduce a Descartes esta pretensión científica en tanto un "volverse como amos y señores de la Naturaleza" (Heidegger, 1994, pp. 135 ss.).

7. Cabe señalar, empero, que, pese a que estas reflexiones heideggerianas parecen traducirse en una crítica a la ciencia misma, no pretenden sino más bien aclarar el sentido íntimo y factual de su ejercicio. En este sentido, tales reflexiones no pueden descartarse sin más aludiendo a que la visión de ciencia que subyace a las consideraciones heideggerianas es fundamentalmente positivista (cf. Siegfried, 1990). A nuestro juicio, el positivismo es un modo como la relación ocupada con el mundo puede realizarse, mas la identificación del presente como la temporalidad propia de la ciencia no es sino el contexto existencial que rinde cuenta de tal modo de

Comte, A. (1898). Discours sur l'esprit positif. Paris: Edition du centenaire d'Auguste Comte.

Descartes, R. (1641/1996). Méditations métaphysiques. Oeuvres, IX: Paris: Vrin.

Einstein, A., Infeld, L. (1938/1961). La física. Aventura del pensamiento. Buenos Aires: Losada.

Elias, N. (1989/2013). Sobre el tiempo. México: Fondo de Cultura Económica.

Fink, E. (1957). Nachdenkliches zur ontologischen Frühgeschichte con Raum-Zeit-Bewegung. La Haya: Martinus Nijhoff.

Galilei, G. (1638/1898). Discorsi e dimostrazioni matematiche intorno a due nuove scienze. Le opere di Galileo Galilei, VIII. Florencia: G. Barbera. investigación positivista, como de cualquier otro, en tanto ciencia, en esencia, es ocupación investigativa del mundo.

8. Se advierte, en este contexto, que las presentes consideraciones se han propuesto realizar una consideración genética del tiempo vulgar, para una reconducción del mismo a sus fundamentos existenciales de carácter temporal. Para una exposición de una fenomenología genética heideggeriana del tiempo remitimos al trabajo de Joan González: Heidegger y los relojes. Dicho trabajo se constituye en un estudio exhaustivo de las consideraciones temporales heideggerianas, pero que, a su vez, se confronta críticamente con las descripciones del tiempo descritas por Heidegger, incluyendo nuevos elementos para su consideración, como es el caso de lo que el autor señala como "la vivencia originaria de la noche" (cf. González, 2008, pp. 41-55).

9. Asimismo, en el contexto del pensar tardío de Heidegger, se hace necesario indagar en la confrontación heideggeriana con la física y su método matemático en el horizonte de la "historia del ser" (Seinsgeschichte) (cf. Vagt, 2012).

10. Esta es la perspectiva que se abre justamente como investigación incluso en el contexto de la polémica frase heideggeriana "la ciencia no piensa", entendida como un llamado a que la ciencia misma finalmente se apropie de su objeto de estudio mediante una aclaración de los conceptos en los que ella se funda (cf. Botavanja, 2008).

García Doncel, M. (1989). El tiempo en la física: de Newton a Einstein. Enrahonar, 15 , pp. 39-59.

González, J. (2008). Heidegger y los relojes. Madrid: Encuentro.

Hacyan, S. (2004). Física y metafísica del espacio y del tiempo. México: Fondo de Cultura Económica.

Heidegger, M. (1987/1994). Zollikoner Seminare. Frankfurt a. M.: V. Klostermann.

Heidegger, M. (1976/1995). Logik: Die Frage nach der Wahrheit. Frankfurt a. M.: V. Klostermann.

Heidegger, M. (1975/1997). Die Grundprobleme der Phänomenologie. Frankfurt a. M: V. Klostermann. 
Heidegger, M. (1954/2000). Die Frage nach der Technik. Vorträge und Aufsätze. Frankfurt a. M: V. Klostermann.

Heidegger, M. (1927/2001). Sein und Zeit Tübingen: Max Niemeyer Verlag.

Heidegger, M. (1977/2005). “Seminar in Le Thor 1969". Seminare. Frankfurt a. M: V. Klostermann.

Husserl, E. (1954/1992). Die Krisis der europäischen Wissenschaften. Hamburg: Felix Meiner Verlag.

Johnson, F. (2011). La crisis de las ciencias: crisis en el conocimiento del mundo. Laguna: Revista de Filosofía, 28, pp. 39-52.
Johnson, F. (2012a). Filosofía y ciencia a partir del pensamiento temprano de Heidegger. Trans/form/açao. Revista de filosofía, 35, 2, pp. 99-120.

Johnson, F. (2012b). La investigación acerca del otro y la filosofía existencial. Actuel Marx/Intervenciones, 12, pp. 45-69.

Johnson, F. (2013). El Dasein caído: esbozo del ámbito fenoménico de la corporalidad humana. Revista de Filosofía Aurora, 36, pp. 287-304.

Mach, E. (1885/1922). Die Analyse der Empfindungen und das Verhältnis des Physischen zum Psychischen. Jena: Gustav Fischer.
Pöggeler, O. (1993). The hermeneutics of the technological world: The Heidegger-Heisenberg dispute. International Journal of Philosophical Studies, 1, 1, pp. 21-48.

Reiser, O. (1926). The problem of time in Science and Philosophy. The Philosophical Review, 35, pp. 236-252.

Seigfried, H. (1990). Autonomy and quantum physics: Nietzsche, Heidegger, and Heisenberg. Philosophy of Science, 57, pp. 619-630.

Vagt, C. (2012). Geschikte Sprünge: Physik und Medium bei Martin Heidegger. Züruch: Diaphanes. 